\title{
The evolution of the oxygen abundance radial gradient in the Milky Way Galaxy disk
}

\author{
Mercedes Mollá ${ }^{1}$, Oscar Cavichia ${ }^{2}$, Roberto D. D. Costa ${ }^{3}$, \\ Walter J. Maciel ${ }^{3}$, Brad Gibson $^{4}$ and Angeles I Díaz ${ }^{5,6}$ \\ ${ }^{1}$ CIEMAT, Avda. Complutense 40, 28040 Madrid, Spain \\ email: mercedes.molla@ciemat.es \\ ${ }^{2}$ Instituto de Física e Química, Universidade Federal de Itajubá, Av. BPS, 1303, 37500-903, \\ Itajubá-MG, Brazil \\ ${ }^{3}$ Instituto de Astronomia, Geofisica e Ciências Atmosféricas, Universidade de Sâo Paulo, \\ 05508-900, Sâo Paulo-SP, Brazil \\ ${ }^{4}$ E.A Milne Centre for Astrophysics, University of Hull, HU6 7RX, United Kingdom \\ ${ }^{5}$ Universidad Autónoma de Madrid, 28049, Madrid, Spain \\ ${ }^{6}$ Astro-UAM, Unidad Asociada CSIC, Universidad Autónoma de Madrid,28049, Madrid, Spain
}

\begin{abstract}
We review the state of our chemical evolution models for spiral and low mass galaxies. We analyze the consequences of using different stellar yields, infall rate laws and star formation prescriptions in the time/redshift evolution of the radial distributions of abundances, and other quantities as star formation rate or gas densities, in the Milky Way Galaxy; In particular we will study the evolution of the oxygen abundance radial gradient analyzing its relation with the ratio SFR/infall. We also compare the results with our old chemical evolution models, cosmological simulations and with the existing data, mainly with the planetary nebulae abundances.
\end{abstract}

Keywords. galaxies: evolution, galaxies: formation, galaxies: spiral, galaxies: abundances, Galaxy: abundances

\section{Introduction}

Chemical elements are mostly created by stellar nucleosynthesis, and eventually ejected and diluted in the interstellar medium when stars die. They are then incorporated into the successive generations of stars. This cycle of formation and death of stars and the subsequent ejection of elements may occur many times, if the star formation rate (SFR) is high, with elemental abundances increasing rapidly in the interstellar medium (ISM); If the SFR is low, the abundances will increase slowly, maintaining low during a long time. This way, elemental abundances give clues about when, how and with which rate stars form, existing a direct link between the evolutionary histories of galaxies or galaxy regions and the abundances measured within them. Chemical evolution models try to understand the metal enrichment process, predicting the elemental abundances expected in each region as depending on different inputs and hypotheses, as the Initial Mass Function (IMF), the star formation law, the existence or not of inflows or outflows of gas, or the stellar nucleosynthesis yields. Once computed a model for a galaxy, it is possible to compare with the observational data and deduce if our assumptions are valid or it is necessary to modify them.

Many numerical chemical evolution models have been presented in the literature from decades ago Lacey \& Fall (1983), Lacey \& Fall (1985), Díaz \& Tosi (1984), Tosi \& Díaz (1985), Matteucci \& Francois (1989), Ferrini et al. (1994). All of them are usually computed to reproduce the Milky Way Galaxy (MWG) and other nearby spiral galaxies data as radial distributions of star formation, gas density, surface density stellar profile 
and the radial gradient of most common elements ( $\mathrm{C}, \mathrm{N}, \mathrm{O}, \mathrm{Fe} . .$.$) , which define the$ present state of our Galaxy. The existence of a radial gradient of abundances in the spiral disks is well known (Henry \& Worthey 1999). This gradient was first observed in the MWG (Shaver et al. 1983) and later in other external galaxies (McCall, Rybski \& Shields 1985, Zaristky, Kennicutt \& Huchra 1994), and it is now well characterized in our local Universe as shown by Sánchez et al. (2014)(S14). However, even fitting the present day data, differences among models appear for other evolutionary times. In particular, the time evolution of the radial gradient of abundances is not the same for all models. Thus, in Díaz \& Tosi (1984), Tosi \& Díaz (1985), Mollá, Díaz \& Tosi (1990) or Chiappini, Matteucci \& Gratton (1997) a flat radial gradient appears in the early times of evolution of a galaxy, steepening with time; on the contrary, in Ferrini et al. (1994), Prantzos \& Aubert (1995), Mollá, Ferrini \& Diaz (1997), Portinari \& Chiosi (2000), and Hou, Prantzos \& Boissier (2000), the gradient is steep, and then it flattens with time. In the first case, a disk is already formed in time zero with an infall of primordial gas which dilutes the enrichment of the disk due to the stars death. In the second type of models, the disk forms out from the gas infalling onto the equatorial plane. Therefore, the evolution of the metallicity gradient seems to be directly related on the formation process of the disk, stressing the importance of this point.

In Mollá, Ferrini \& Diaz (1997) we analyzed the existing data for MWG about this question: planetary nebulae (PN) abundances, globular and open clusters metallicities and stellar abundances. Each data set has his own problems to determine the time evolution of the radial gradient. The PN are useful to estimate the radial gradient of oxygen, an element not modified by the nucleosynthesis in the progenitors stars of PN. The early data analyzing this question (Maciel, Costa \& Uchida 2003) showed a radial gradient steeper than the one for the present time, although, due to error bars, the observational points mixed with the HII regions data in the plot of $\mathrm{O} / \mathrm{H}$ vs galactocentric radius $R$. The open clusters problems are derived of the necessary classification in thin or thick disk or even halo populations before their use in a radial distribution of metallicity. Furthermore, the ages determination, coming from a fit of their spectra to stellar models, which depend on metallicity and on age simultaneously, added to differences depending on the use of LTE or NLTE models, may be uncertain. The estimate of age and metallicity of globular clusters (GC) seems less problematic since there was assumed that they form as a single stellar population, with a single burst of star formation $\dagger$ and, consequently, to obtain only an age and a metallicity for all the stars of a GC is more precise. Based on these GCs data, we concluded that the radial gradient of abundances in MWG was steeper in the past, and it has been flattening with time until arriving to the present value. However, these old objects may also be part of the thick disk or the halo populations. As for the open clusters, the kinematic information is essential to determine their group and, therefore, to know if the corresponding data may be included in the study of the time evolution of the radial distribution of disk abundances.

In Mollá \& Díaz (2005)-hereinafter MD05-, we show 440 chemical evolution models, for 44 different galaxy masses and 10 possible star formation efficiencies in each one. From a MWG-like model, we obtained the evolution of the radial gradient of oxygen abundances, finding a gradient of $\sim-0.2 \mathrm{dex} \mathrm{kpc}^{-1}$ for $z=2$ which flattens with time until reaching the present time value $\sim-0.05-0.06 \mathrm{dex} \mathrm{kpc}{ }^{-1}$. This behavior was in agreement with the cosmological simulations results from Pilkington et al. (2012) and also with the Yuan et al. (2011) value found at $z \sim 1.5$. However, it is necessary to take into account that this gradient was calculated with the $\mathrm{O}$ abundances of all radial regions

$\dagger$ Recent works conclude that there are at least two stellar generations in these old objects. 
computed in our model (which arrived until $25 \mathrm{kpc}$ ). In fact, the optical radius of MWG is $\sim 13-15 \mathrm{kpc}$, and, therefore, to include the regions out of a given radius might be considered as inadequate, since there thick or halo populations may being mixed with the thin disk. Taking into account that these outer regions of the galaxy are not evolved, this implies a steepening of the radial gradient, more strongly for the highest redshift since the parts not evolved of the disk will be more inner. If we only consider the regions within the optical radius to compute the radial gradient, the evolution of this last one along the redshift is smoother, with a value $\sim-0.06 \mathrm{dex} \mathrm{kpc}^{-1}$ for all redshifts.

We have now revised our chemical evolution models to include more realistic and updated inputs in our code. In this work we analyze the corresponding results for our new MWG model, used to calibrating the model grid. We study in particular the evolution of the radial gradient of oxygen, searching for the possible differences with the old model from MD05 and checking if the new prescriptions related on stellar yields, star formation or infall rate have modified the behavior of this gradient. We also compare the results with the most recent data in the literature.

\section{Abundances in the MWG}

In the recent years, new sets of data have been published, as consequence of better instruments, techniques and telescopes. Anders, Chiappini \& Minchev (2016) give a summary of the observations (see their Fig.5), referring to PN, open clusters, and stars from MWG in order to analyze the evolution of the radial gradient of metallicity along the time. The problem is that each type of objects shows a different evolution. Thus, PNs which gave a steep radial gradient for a time $\sim 8$ Gyr compared with the one corresponding to the present time given by HII regions, give now, following the most recent findings, a flat evolution along the time; that is, the radial gradient for $\mathrm{O}$ is basically the same from 5-6 Gyr ago until now (Maciel \& Costa 2013, M13), or even has steepen slightly on time (Stanghellini \& Haywood 2010, S10). A radial gradient constant along the time is also obtained by Magrini et al. (2016) for M33, M31, M81 and NGC300, comparing their radial distributions of oxygen abundances given by HII regions and PN, finding no evidences of evolution of the radial gradient with time. The PN give precise measurements of abundances since they are very bright, allowing to observe them even being far away. However, to estimate their masses (an in consequence their ages) and their distances is not an easy task, as explained in Cavichia, Costa, \& Maciel (2011), which makes the Magrini et al. (2016) constraint not totally certain. From open clusters data, practically all of works, as Friel et al. (2002), Chen, Hou, \& Wang (2003), Magrini et al. (2009), Frinchaboy et al. (2013), Cunha et al. (2016), arrive to a similar conclusion: the radial gradient of metallicity was steeper in the past than now. The number of points of the complete sample in these works is usually small and, therefore, each defined bin of age is wide with a handful of points. Finally, stellar abundances, as given Nordström et al. (2004), Casagrande et al. (2011), Bergemann et al. (2014), Xiang et al. (2015), show a flattening of the radial gradient. However, the oldest age bin (around 12-13 Gyr old) has a radial gradient zero. It means that the radial gradient started flat, then it did steeper, and after it flattened again. Anders, Chiappini \& Minchev (2016) do not agree with these results, obtaining a radial gradient steepening continuously with time. Looking at these data in detail, we find some problems for the precise determination of the radial gradient for the old ages bin. Bergemann et al. (2014) give the metallicity radial distribution as the Iron abundance dividing their sample in different stellar ages, with a radial range for the whole sample of $\sim 4 \mathrm{kpc}$ (actually not very wide), but the oldest stars are located in a reduced region around the Solar vicinity, with a radial range 

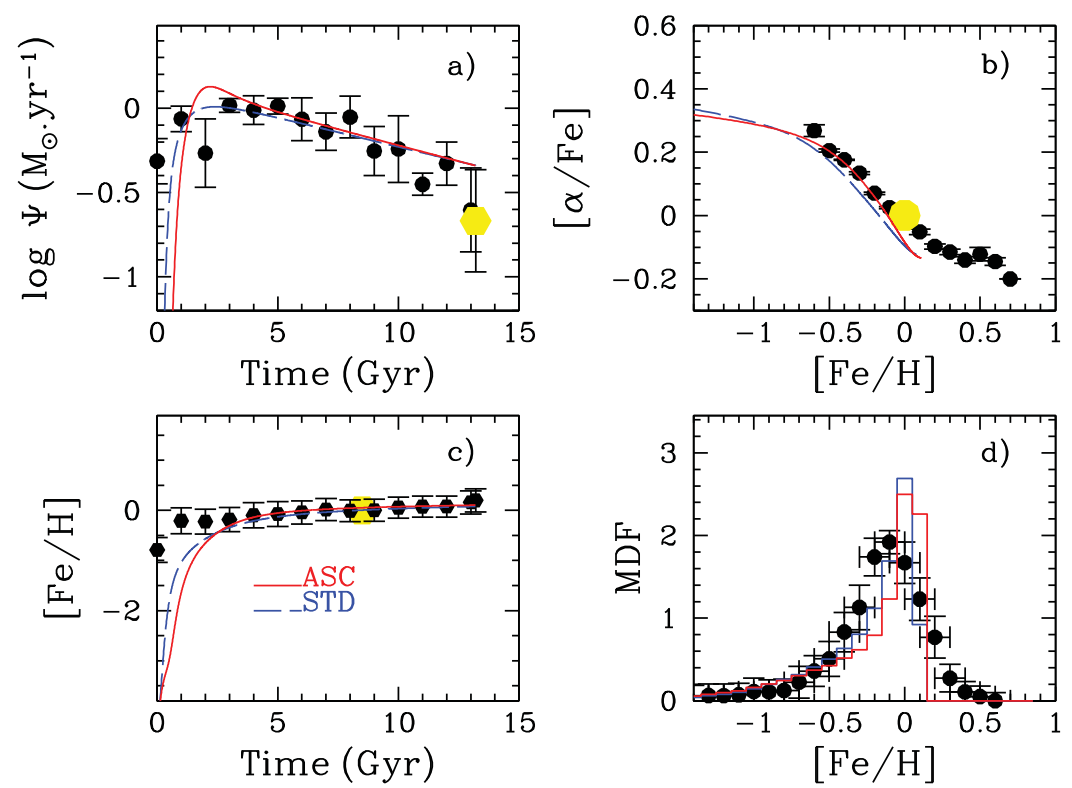

Figure 1. Solar Region evolution: a) SFR as a function of time; b) $[\alpha / F e]$ as a function of $[\mathrm{Fe} / \mathrm{H}]$; c) $[\mathrm{Fe} / \mathrm{H}]$ as a function of time; and d) The metallicity distribution function. In all cases the blue and red lines represent the STD and ASC models, as labelled, while the black dots represent the Solar Region data as compiled in Mollá et al. (2015). The yellow large dot is the value of the SFR at the present time in a), the Solar abundance in b) and c), located in the time when the Sun formed in this last panel.

of $\sim 1 \mathrm{kpc}$. It is impossible to estimate a gradient with such a short radial range. This problem for the oldest age bin also occurs in Xiang et al. (2015), where the radial range reduces from the $3-4 \mathrm{kpc}$ for the youngest bin to $1-1.5 \mathrm{kpc}$ for the oldest one. For the CoRoGee sample from Anders, Chiappini \& Minchev (2016) the total sample has a good radial range from $4 \mathrm{kpc}$ to $14 \mathrm{kpc}$, but the oldest bin has a range much shorter, from 8 to $12 \mathrm{kpc}$. This produces a cloud of points around $\sim 9 \mathrm{kpc}$ of galactocentric distance, which does not allow the computation of a precise radial gradient.

\section{Multiphase Chemical Evolution models}

Our basic model is well described in Ferrini et al. (1994), Mollá \& Díaz (2005). We start with a spherical protogalaxy with a dynamical mass distribution given by the rotation curve. This initial gas falls onto the equatorial plane forming out the disk. In the halo the star formation follows a Schmid-Kennicutt law, while in the disk molecular clouds form first, and then stars are created from cloud-cloud collisions and from the interaction of massive stars with the surrounding clouds. Including the star mean lifetime and the stellar yields, we compute abundances for 15 elements until Fe, being this element mostly created by the Supernova type Ia yields from Iwamoto et al. (1999). In this standard model (STD), the star and molecular cloud formation are calculated with two efficiencies for each galaxy model, which we vary simultaneously with values among 0 and 1 .

We have now an updated grid of models (Mollá et al. in preparation), where we use the stellar yields from Gavilán, Buell \& Mollá (2005), Gavilán, Mollá \& Buell (2006), Limongi \& Chieffi (2003), Chieffi \& Limongi (2004) and the IMF from Kroupa (2002), selected as the best combination of stellar yields and IMF from 144 models in Mollá et al. (2015). We have also revised the infall rates of gas, calculated to create disks as observed following 


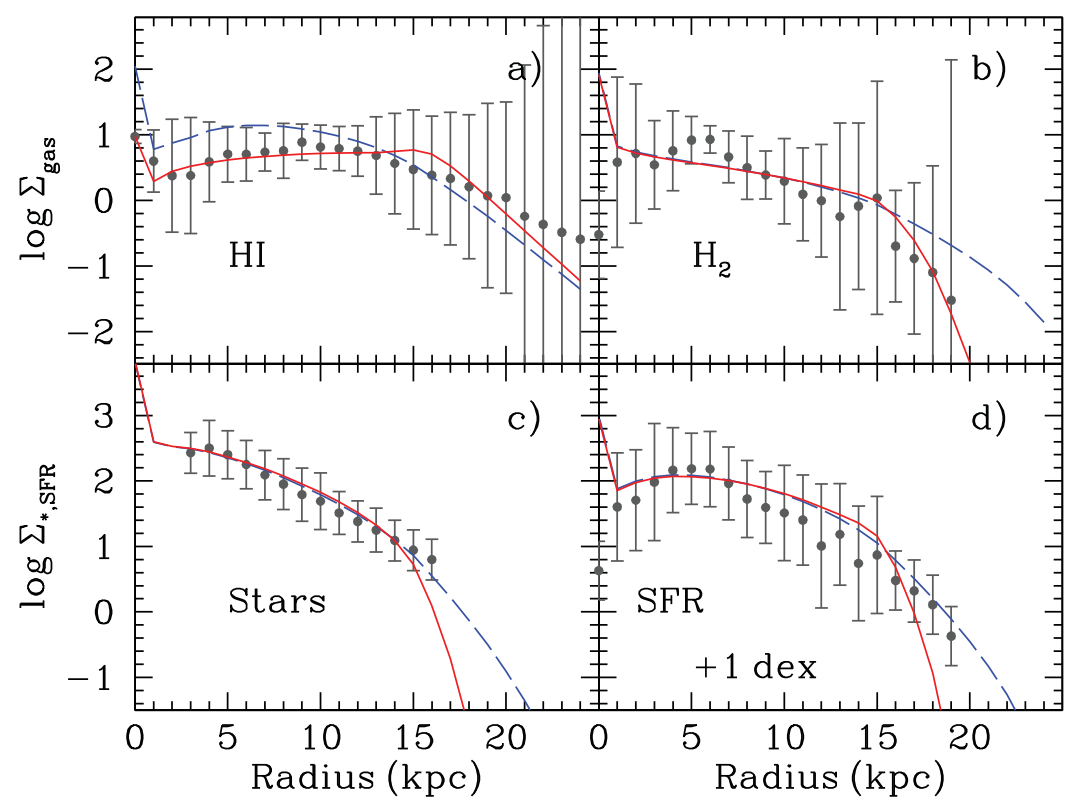

Figure 2. Radial distributions of surface density for: a) Diffuse gas; b) Molecular gas; c) Stellar profile; and d) SFR. In all cases the blue and red lines represent the STD and ASC models while the black dots represent the MWG data as compiled in the Appendix from Mollá et al. (2015).

Salucci et al. (2007). Details are given in Mollá et al. (2016). Moreover, we now follow the prescription by Ascasibar et al. (in preparation) to form molecular clouds from the diffuse gas. This model (ASC) is compared with our standard model (STD). The details are given in Mollá et al. (2017).

For a MWG-like model, we use a dynamical mass of $10^{12} \mathrm{M}_{\odot}$, able to create a disk of $\sim 710^{10} \mathrm{M}_{\odot}$. In Fig. 1 we represent the evolution of the region located a $R=8 \mathrm{kpc}$ as function of time in panels a) and c), and as function of the metallicity $[\mathrm{Fe} / \mathrm{H}]$ in panels b) and d), compared with the Solar Region data. In Fig. 2 we draw the surface densities of $\mathrm{HI}, \mathrm{H}_{2}$, stars and SFR for the disk component, compared with the MWG data. We compare both STD and ASC models, finding that basically both fit the data within errors, with differences only in the outer regions with a more extended disk for STD.

\section{Results: the time evolution of oxygen radial gradient}

In Fig. 3 we show the radial distribution for $\mathrm{O} / \mathrm{H}$ as given by STD (top panels) and ASC (bottom panels) prescriptions, for different redshifts as labelled in the top right panel. This way, we may check the effect of the star formation law on the evolution of the radial gradient. The distributions shows a sharp cut of the disk in ASC while this is smoother in STD, with a more extended disk, as said before. This is due to the effect of threshold of ASC, since the necessary density or metallicity must be reached to create molecular clouds. In left panels we show these distributions as a function of the galactocentric distance $R$ (in $\mathrm{kpc}$ ). In right panels we show the same distributions as a function of the normalized radius $R / R_{e f f}$. It is clear that the slope of the distribution within the optical disk, defined as $R \sim 3 R_{e f f}(\sim 15 \mathrm{kpc}$ for the STD model at $z=0)$, is almost the same for all redshifts, as shown in right panels. We must remember that $R_{e f f}$ changes with redshift: the stellar disk increases their size when stars form in an inside-out scenario; at the early times stars form only in the inner disk. In the STD model, $R_{e f f}$ is 


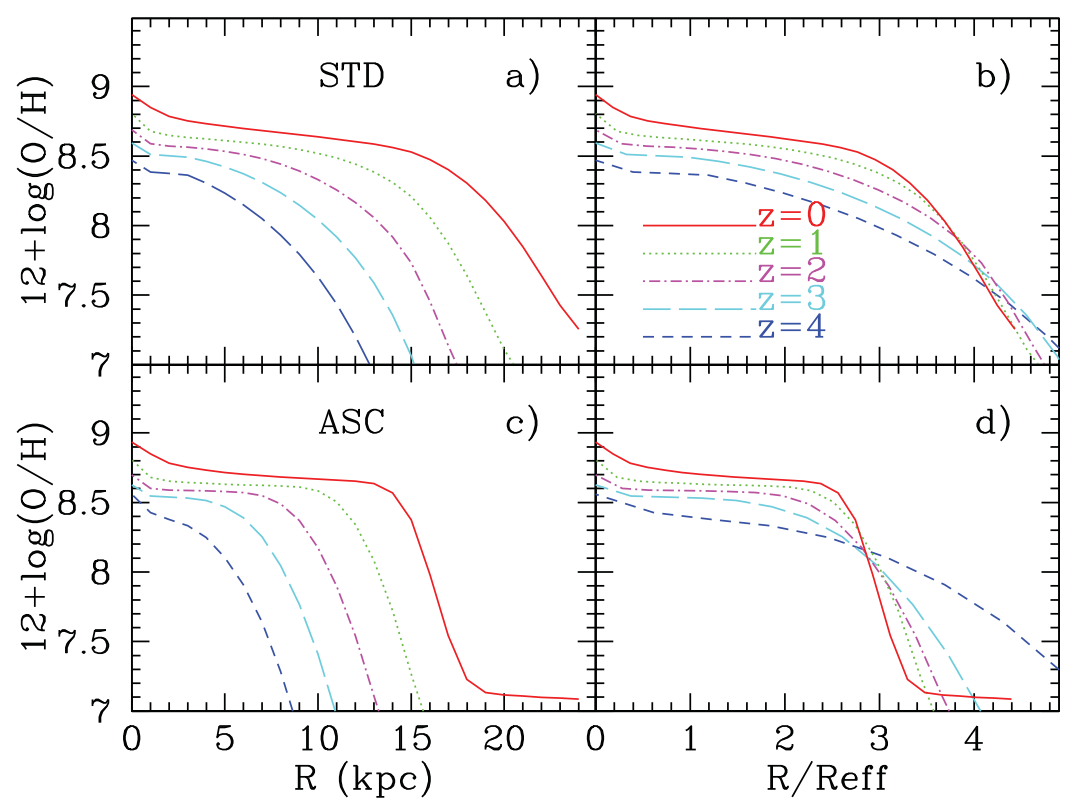

Figure 3. Radial distributions of the $12+\log (\mathrm{O} / \mathrm{H})$ abundances for different redshifts as labelled. Left panels as function of the galactocentric distance (in $\mathrm{kpc}$ ) and right panels as function of the normalized radius $R / R_{e f f}$. Top panels represent the evolution of STD, while the bottom ones refer to ASC.

around $5 \mathrm{kpc}$ for the present time, but it is $\sim 2.5 \mathrm{kpc}$ at $z=4$. However, $R_{\text {eff }}$ increases very slowly in ASC model, being $R_{\text {eff }} \sim 1.6 \mathrm{kpc}$ at $z=4$. Thus, at this redshift, the optical radius would be around $\sim 7 \mathrm{kpc}$ in STD model, but it only arrives to $\sim 4.5 \mathrm{kpc}$ in ASC. Therefore, it seems quite evident that the slope of the radial gradient is basically the same for all redshifts when is measured within the optical radius, where we must define a radial gradient of the disk oxygen abundance. If a radial gradient different than the one of the present time is obtained at other time/redshift, it implies that it is being measured with regions out of the optical disk, probably with the thick disk, or of the halo, not unexpected since the phases of the disk formation occur at $z>2.5$.

We summarize our results in Fig. 4, with the evolution along redshift of the $\mathrm{O} / \mathrm{H}$ radial gradient, measured as dex $\mathrm{kpc}^{-1}$ in the top panels and as dex $\mathrm{R}_{\text {eff }}^{-1}$ in the bottom ones. In left panels the gradient is calculated using the whole radial range that we run our MWG model, while in the right ones we use only regions within the optical radius. Top panel results are compared with our old model MD05, cosmological simulations by Gibson et al. (2013)(G13) and recent data as labelled, including M13 from PN and also Henry et al. (2010), Rupke, Kewley \& Chiel (2010), Cresci et al. (2010), Queyrel et al. (2012), and Jones et al. (2013) as H10,R10, C10, Q12 and J13, respectively, for high redshift galaxies.

\section{Conclusions}

The main conclusions can be summarized as:

(a) The radial range where the least-squares straight line fit is done is crucial to determine the value of the abundance radial gradient. 


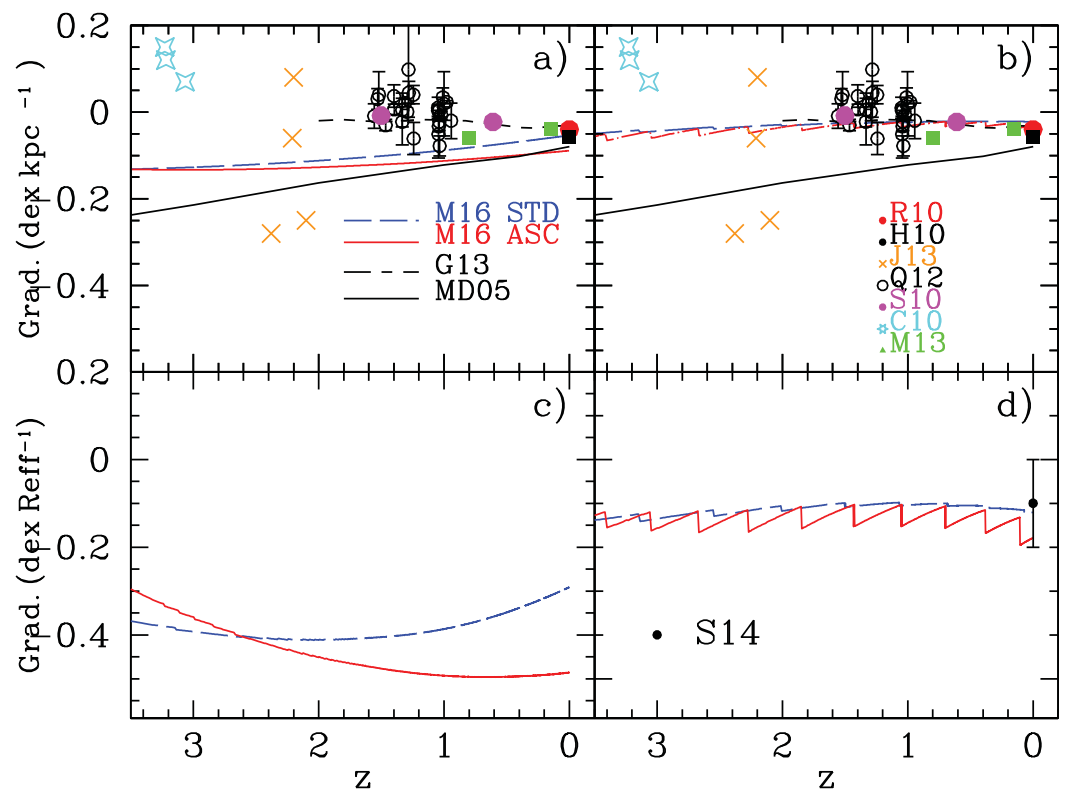

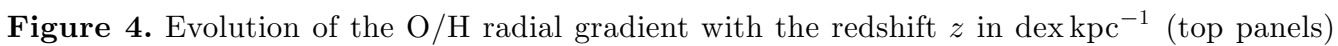
and dex $R_{e f f}^{-1}$ (bottom panels). In a) and c) calculated with the whole radial range. In b) and d) calculated with $R \leqslant 3 R_{e f f}$. In top panels, data are from H10, R10, S10, C10, Q12, J13 and M13. Models STD and ASC are our new models (M16) -this work and Mollá et al. (2017)-, our old model MD05 and simulation G13. In panel d) the present day value corresponds to the average gradient found by S14.

(b) Our MWG models produce a smooth evolution of the $\mathrm{O}$ abundances radial gradient with a value of $-0.15 \mathrm{dex} \mathrm{kpc}^{-1}$ at $z=3.5$ arriving to $-0.05 \mathrm{dex} \mathrm{kpc}^{-1}$ at $z=0$ when the whole radial range is used.

(c) The radial gradient has a value around $\sim-0.05 \mathrm{dex} \mathrm{kpc} c^{-1}$ for $z \leqslant 3.5$ when it is measured within the optical radius (variable with $z$ ).

(d) The radial gradient computed by our new models is flatter than the obtained in MD05 as consequence of the new infall rates, smoother than the old ones.

(e) If the radial gradient is measured as a function of a normalized radius $\left(R / R_{e f f}\right)$, differences arise between STD and ASC proceeding from the different grow of size of the stellar disk in both models (even with the same infall rates), if all radial regions are used.

$(f)$ If the radial gradient is computed within the optical radius, as a function of a normalized radius $\left(R / R_{e f f}\right)$, it has a constant value of $\sim-0.15 \operatorname{dex} \mathrm{R}_{e f f}^{-1}$ for $z \leqslant 3.5$, which agrees with Sánchez et al. (2014) result for the CALIFA local galaxies.

\section{References}

Anders, F., Chiappini, C., Minchev, I., et al. 2016, A\&A, submitted (arXiv:1608.04951)

Bergemann M., et al., 2014, A\&A A, 565, A89

Casagrande, L., Schönrich R., Asplund M., Cassisi S., et al., 2011, A\&AA, 530, A138

Cavichia O., Costa R. D. D., \& Maciel W. J., 2011, $R M x A A, 47,49$

Chen L., Hou J. L., \& Wang J. J., 2003, AJ, 125, 1397

Chiappini, C., Matteucci, F., \& Gratton, R. 1997, ApJ, 477, 765

Chieffi A. \& Limongi M., 2004, ApJ, 608, 405

Cresci, G., Mannucci, F., Maiolino, R., et al. 2010, Nature, 467, 811 (C10)

Costa, R. D. D., Cavichia, O., \& Maciel, W. J., 2013, IAUS, 289, 375 
Cunha, K., Frinchaboy, P. M., Souto, D., et al. 2016, Astronomische Nachrichten, 337, 922

Diaz, A. I. \& Tosi, M., 1984, MNRAS, 208, 365

Ferrini, F., Molla, M., Pardi, M. C., \& Diaz, A. I., 1994, ApJ, 427, 745

Friel, E. D., Janes, K. A., Tavarez, M., Scott, J., Katsanis, R., et al., 2002, AJ, 124, 2693

Frinchaboy, P. M., et al., 2013, ApJ, 777, L1

Gavilán, M., Buell, J. F., \& Mollá, M., 2005, A\&SA, 432, 861

Gavilán, M., Mollá, M., \& Buell, J. F., 2006, A\& A, 450, 509

Gibson, B. K., Pilkington, K., Brook, C. B., Stinson, G. S., \& Bailin, J. 2013, A\&3A, 554, A47 (G13)

Henry, R. B. C. \& Worthey, G. 1999, PASP, 111, 919

Henry, R. B. C., Kwitter, K. B., Jaskot, A. E., et al. 2010, ApJ, 724, 748 (H10)

Hou, J. L., Prantzos, N., \& Boissier, S. 2000, A\&A, 362, 921

Iwamoto, K., Brachwitz, F., Nomoto, K., et al. 1999, ApJS, 125, 439

Jones, T., Ellis, R. S., Richard, J., \& Jullo, E. 2013, ApJ, 765, 48 (J13)

Kroupa, P., 2002, Sci, 295, 82

Lacey, C. G. \& Fall, S. M. 1983, MNRAS, 204, 791

Lacey, C. G. \& Fall, S. M. 1985, ApJ, 290, 154

Limongi, M. \& Chieffi, A., 2003, ApJ, 592, 404

Maciel, W. J., Costa, R. D. D., \& Uchida, M. M. M., 2003, A\&A, 397, 667

Maciel, W. J. \& Costa, R. D. D. 2013, RMxA\&Ap, 49, 333 (M13)

Magrini, L., Sestito, P., Randich, S., \& Galli, D. 2009, A\&A, 494, 95

Magrini, L., Coccato, L., Stanghellini, L., Casasola, V., \& Galli, D. 2016, A\&A A, 588, A91

Matteucci, F., \& Francois, P. ,1989, MNRAS, 239, 885

McCall, M. L., Rybski, P. M., \& Shields, G. A. 1985, ApJS, 57, 1

Mollá, M., Díaz, A. I., \& Tosi, M., 1990, in Chemical and dynamical evolution of Galaxies, eds.

F. Ferrini, J. Franco, \& F. Matteucci, ETS Editrice (PISA), 577

Mollá, M., Ferrini, F., \& Diaz, A. I. 1997, ApJ, 475, 519

Mollá, M. \& Díaz, A. I. 2005, MNRAS, 358, 521 (MD05)

Mollá, M., Cavichia O., Gavilán M., \& Gibson B. K. 2015, MNRAS, 451, 3693

Mollá, M., Díaz, A. I., Gibson, B. K., et al., 2016, MNRAS, 462, 1329

Mollá, M., Ascasibar, Y., Díaz, A. I., \& Gibson, B. K., 2017, MNRAS, submitted (M16)

Nordström B., et al., 2004, A\&A, 418, 989

Pilkington, K., Few, C. G., Gibson, B. K., et al. 2012, Aछ A, 540, A56

Portinari, L. \& Chiosi, C. 2000, A\&A, 355, 929

Prantzos, N. \& Aubert, O. 1995, A\& A, 302, 69

Queyrel, J., Contini, T., Kissler-Patig, M., et al. 2012, A\&AA, 539, A93 (Q12)

Rupke, D. S. N., Kewley, L. J., \& Chien, L.-H. 2010, ApJ, 723, 1255 (R10)

Salucci, P., Lapi, A., Tonini, C., Gentile, G., et al.., 2007, MNRAS, 378, 41

Sánchez, S. F., Rosales-Ortega, F. F., Iglesias-Páramo, J., et al. 2014, A\&AA, 563, A49 (S14)

Shaver, P. A., McGee, R. X., Newton, L. M., Danks, A. C., et al. 1983, MNRAS, 204, 53

Stanghellini, L. \& Haywood, M. 2010, ApJ, 714, 1096 (S10)

Tosi, M. \& Díaz, A. I. 1985, MNRAS 217, 571

Xiang, M.-S., Liu, X.-W., Yuan, H.-B., et al. 2015, Research in Astronomy and Astrophysics, 15,1209

Yuan, T.-T., Kewley, L. J., Swinbank, A. M., et al. . 2011, ApJL, 732, L14

Zaritsky, D., Kennicutt, R. C., Jr., \& Huchra, J. P. 1994, ApJ, 420, 87

\section{Discussion}

Hensler: Part of the produced oxygen will be stored in the halo of galaxies (COS halo survey) or lost from it due to interactions with the environment. Its contribution to the oxygen enhancement depends on the time scale of this backflow cycle. How can you incorporate this effect into your models? 
Q: UV absorption lines are used to measure the abundances of gas at large radii from spiral galaxies. Could you please summarize the results and how they compare to theoretical predictions of metal enrichment? 